\title{
Interferon graft dysfunction: a final chapter for interferon and hepatitis $\mathrm{C}$
}

\author{
Julie A. Thompson · John R. Lake
}

Received: 29 January 2014/ Accepted: 27 March 2014/Published online: 6 April 2014

(C) Asian Pacific Association for the Study of the Liver 2014

\begin{abstract}
Alpha-interferon (IFN) has been the backbone of treatments for the hepatitis $\mathrm{C}$ virus (HCV) for a quarter of a century. HCV universally recurs after liver transplantation (LT), and there is more rapid progression of fibrosis, estimated to be twice that of pre-LT HCV. The chance of developing cirrhosis at 5 years has been reported to range from 15 to $50 \%$, depending on the reference. Mortality in patients transplanted for $\mathrm{HCV}$ is the worst among the various disease indications for LT. Because of increased morbidity and mortality in this population, there has been a logical push to improve outcomes by attempting to aggressively treat post-LT HCV. However, sustained virological response (SVR) rates with IFN-based post-LT treatment have been relatively poor, and there have been, heretofore, no other effective treatments for HCV. Moreover, there are risks associated with post-LT IFN-based HCV treatment.
\end{abstract}

In this issue of Hepatology International, Ikegami and colleagues from Kyushu University describe interferoninduced graft dysfunction (IGD) in a series of 80 patients who received pegylated-IFN (Peg-IFN) after living donor LT [1]. Their goal was to identify common characteristics and management strategies for this potentially morbid condition. Patients were treated for HCV if they had recurrent $\mathrm{HCV}$ with abnormal liver tests and histologic evidence of hepatitis. Most patients had treatment initiated at 6 months post-LT. These authors chose Peg-IFN $\alpha-2 b$ with ribavirin for 48 weeks as primary treatment but switched patients to Peg-IFN $\alpha-2 a$ if HCV RNA levels did

J. A. Thompson · J. R. Lake ( $₫)$

University of Minnesota, Minneapolis, MN, USA

e-mail: lakex009@tc.umn.edu

J. A. Thompson

e-mail: thom0235@umn.edu not decrease satisfactorily. Their reasoning for this conversion was based on the longer half-life of Peg-IFN $\alpha-2 a$ and other studies suggesting that this strategy may increase the chance for SVR [2].

They defined IGD as "graft dysfunction during or after interferon treatment for HCV. The pathological diagnoses included plasma cell hepatitis ( $\mathrm{PCH})$, acute cellular rejection (ACR), or chronic rejection (CR), or a combination of these findings." They did not discuss or compare pathological or laboratory abnormalities in their LT patients who did not receive interferon, with or without HCV.

They defined ACR as mixed lymphocytic aggregates with bile duct damage and vascular endotheliitis, a standard definition. PCH was identified by the presence of plasma cell aggregates without bile duct damage or endotheliitis. Plasma cell aggregates along with bile duct damage or endotheliitis was considered PCH + ACR. CR was defined as having bile duct loss, Glissonean or central venous fibrosis progression without cellularity, portal venous peliosis and foamy hepatic arterial cells.

Eight patients experienced IGD: one with ACR, two with $\mathrm{PCH}$, three with $\mathrm{PCH}+\mathrm{ACR}$ and two with $\mathrm{CR}$ (initially diagnosed as ACR and with $\mathrm{PCH}+\mathrm{ACR}$ ). Six of these patients were treated with Peg-IFN $\alpha$ 2a, and four of them developed IGD after the switch from $2 b$ to $2 a$. When IGD was identified, IFN treatment was stopped and immunosuppression increased. The patients who manifested CR experienced graft loss. Of those without CR, five of them achieved SVR.

Treatment with Peg-IFN $\alpha$ 2a was the only factor found to significantly contribute to IGD. Patients with IGD had lower 5-year graft survival compared to those without (71 vs. $93 \%$ ).

While the number of patients studied was small, this study reminds us of the controversial classification of the 
pathological entities that affect liver allografts. The pathophysiology of IGD has only recently been described and is still not well understood. These authors considered PCH, $\mathrm{ACR}$ and $\mathrm{CR}$, all diagnoses to be classified under the umbrella of IGD, an immune-mediated hepatitis. Whether this is appropriate remains to be determined.

Successful post-LT treatment of HCV has been shown to improve survival in $\mathrm{HCV}$-infected LT patients, but there are risks. IFN affects both the innate and adaptive immune systems, and a variety of immune-related phenomena have been described with its use [3]. The use of exogenous IFN has been reported to lead to autoimmune hepatitis and induce other types of autoimmune disease [4]. Interferon has been suggested to provoke rejection, acute or chronic, in renal allografts and has been generally regarded as relatively contraindicated in renal transplant patients.

The role of IFN in precipitating rejection in LT patients is not clear and not always recognized. In many studies, patients were not biopsied during treatment, and, even so, the diagnosis of rejection may be difficult to differentiate from recurrent $\mathrm{HCV}$.

ACR associated with IFN therapy appears to be relatively uncommon after LT. Estimates of ACR occurrence with IFN-based treatment vary from 0 to $35 \%$ [5]. The variation in the reported incidence of ACR reflects a number of factors including nonuniformity in obtaining biopsies, IFN treatments (pegylated vs. non-pegylated) and the baseline immunosuppression regimens. ACR typically manifests with elevated transaminases, but the diagnosis requires liver biopsy. The Banff Working Group developed a consensus system for ACR in 1997: (1) a mixed, but predominantly mononuclear portal inflammation, containing blastic (activated) lymphocytes, neutrophils, and frequently eosinophils; (2) bile duct inflammation/damage; (3) phlebitis of portal veins or terminal hepatic venules [6]. This definition works well for acute rejection during the early posttransplant period, but late rejection can be more variable in its presentation.

De novo "autoimmune" hepatitis has been well described in LT recipients. It has been described as an alloimmune hepatitis with lymphoplasmacytic infiltrates. It is still debated whether this is a form of ACR or CR, or is a true alloimmune hepatitis. In 2008, Fiel et al. [7] described a related entity they termed "plasma cell hepatitis" after observing the common finding of plasma cell-rich infiltrates in liver allografts biopsies. A histologic scoring system was developed to assist with the diagnosis and included the presence of numerous plasma cells, often in sheets or clusters, and centrilobular necrosis. Patients affected with PCH had dense plasma cell infiltrates and were at greater risk for graft loss. Over half of these patients had experienced a previous episode of ACR. These authors concluded that $\mathrm{PCH}$ is a histologic variant of rejection because there was a frequent association with development of PCH and suboptimal immunosuppression preceding acute rejection as well as better outcomes when immunosuppression was increased. However, the pathologic processes leading to $\mathrm{PCH}$ are still poorly understood.

Relevant to the current publication, $\mathrm{PCH}$ can also occur in the setting of interferon-related graft injury. Because overlap exists with recurrent $\mathrm{HCV}$, de novo autoimmune hepatitis and ACR, the diagnosis of $\mathrm{PCH}$ can be difficult to make $[8,9]$. It is still unclear how to characterize PCH in the setting of interferon-based therapy: as its own entity, as a manifestation of treated hepatitis $\mathrm{C}$ or as a variant of rejection.

In a case-control series, Levitsky et al. [10] reported that PCH was the most common manifestation of IGD. They concluded that $\mathrm{PCH}$ was underrecognized and frequently reported as recurrent hepatitis $\mathrm{C}$ or a different type of rejection. They also found that use of Peg-IFN 2a was a risk factor for developing IGD and that IGD was associated with worse outcomes.

The understanding of IFN effects in liver allografts is soon to be of historical interest. Going forward, we will have available highly effective direct acting antivirals against $\mathrm{HCV}$ that will be used without interferon and will likely not lead to the immunologic phenomena described in the current publication. The story of interferon and its role as an antiviral began before hepatitis $\mathrm{C}$ had even been identified. In 1957, Isaacs and Lindenmann found that interferon, a naturally occurring substance, could act as an antiviral agent [11]. It was given the name to reflect its ability to "interfere" with replication of viruses. It would not be until 34 years after the discovery of interferons that they would be available for HCV treatment. Even with its myriad and often severe side effects and dreaded reputation, it has been effective in treating perhaps hundreds of thousands of cases of both pre- and post-LT HCV. The side effects and morbidity associated with IFN have been the topic of many reports and have often limited the safe treatment of LT patients. IGD, an immune-mediated process that has only recently been described, is still not well understood, and the entities that manifest as a part of it, $\mathrm{PCH}, \mathrm{ACR}$ and $\mathrm{CR}$, will continue to be debated after the book closes on the use of IFN to treat HCV.

\section{References}

1. Ikegami $\mathrm{T}$, Wang $\mathrm{H}$, Yoshizumi $\mathrm{T}$, Toshima $\mathrm{T}$, Aishima $\mathrm{S}$, Fukuhara F, et al. Strategies to treat interferon-induced graft dysfunction after living donor liver transplantation for hepatitis C. Hepatol Int. 2014. doi:10.1007/s12072-013-9496-2

2. Herrine SK, Brown RS Jr, Bernstein DE, Ondovik MS, Lentz E, Te H. Peg-interferon alpha-2a combination therapies I chronic 
hepatitis $\mathrm{C}$ patients who relapsed after or had viral breakthrough on therapy with standard interferon alpha- $2 \mathrm{~b}$ plus ribavirin: a pilot study of efficacy and safety. Dig Dis Sci. 2005;50:719-726

3. Feld JJ, Hoofnagle JH. Mechanism of action of interferon and ribavirin in treatment of hepatitis C. Nature. 2005;436:967-972

4. Ganne-Carrie N, Medini A, Coderc E, Seror O, Christidis C, Grimbert $\mathrm{S}$, et al. Latent autoimmune thyroiditis in untreated patients with HCV chronic hepatitis: a case-control study. J Autoimmun. 2000;14:189-193

5. Watt K, Veldt B, Charlton M. A practical guide to the management of $\mathrm{HCV}$ infection following liver transplantation. Am J Transplant. 2009;9:1707-1713

6. Banff schema for grading liver allograft rejection: an international consensus document. Hepatology. 1997;25:658-663

7. Fiel MI, Agarwal K, Stanca C, Elhajj N, Kontorinis N, Thung SN, et al. Posttransplant plasma cell hepatitis (de novo autoimmune hepatitis) is a variant of rejection and may lead to a negative outcome in patients with hepatitis $\mathrm{C}$ virus. Liver Transpl. 2008;14:861-871

8. Khettry U, Huang WY, Simpson MA, Pomfret EA, Pomposelli JJ, Lewis WD, et al. Patterns of recurrent hepatitis C after liver transplantation in a recent cohort of patients. Hum Pathol. 2007;38:443-452

9. Tun HW, Krishna M, Menke DM. Hepatitis C-related posttransplant plasma cell proliferative disorder with hepatitis $\mathrm{C}$ virus in neoplastic plasma cells: a new posttransplant disease entity? Transpl Proc. 2004;36:2692-2696

10. Levitsky J, Fiel MI, Norvell JP, Wang E, Watt KD, Curry MP, et al. Risk for immune-mediated graft dysfunction in liver transplant recipients with recurrent $\mathrm{HCV}$ infection treated with pegylated interferon. Gastroenterology. 2012;142(1132-1139):e1

11. Isaacs A, Lindenmann J. Virus interference. I. The interferon. Proc R Soc. 1957;B147:258-267 